\title{
Subretinal Angiostrongyliasis: A Case Report
}

\section{Sugamon Koohasawad}

Department of Ophthalmology, Neurological Institute of Thailand, Bangkok, Thailand
Correspondence: Sugamon Koohasawad Department of Ophthalmology, Neurological Institute of Thailand, 312 Ratchawithi Road, Thung Phaya Thai, Ratchathewi, Bangkok, 10400, Thailand Tel +6623069899

Fax +6623545357

Email sugamon.koo@gmail.com
Purpose: To report the patient with subretinal Angiostrongylus larva.

Methods: Retrospective, observational case report.

Results: A 47-year-old Thai woman had eosinophilic meningitis. One week after the onset of a headache, blurred vision developed in her right eye. Ocular examination of the right eye showed Angiostrongylus larva in the subretinal space in the inferotemporal quadrant and macular edema. Although serum analysis for Angiostrongylus was negative, the larva can be identified by its characteristic appearance. Blood eosinophilia and cerebrospinal fluid eosinophilia were presented. Focal laser photocoagulation was applied to the retina, and the patient also received anti-helminthic and oral corticosteroid drugs. The vision did not recover. Ultimately, the retina became atrophic. The dead larva remained at the same site at which it was observed. No further larval migration occurred after treatment.

Conclusion: Angiostrongylus larva can damage the retinal layer despite its eradication and no further migration. After its death, the inflammation persisted either as the result of toxin secretion or an immune responsiveness. Administration of local anti-inflammatory drugs such as corticosteroids or any anti-vascular endothelial growth factor injection should be considered.

Keywords: subretinal Angiostrongylus larva, ocular angiostrongyliasis, intraocular angiostrongyliasis, Angiostrongylus canthnensis, intraocular parasite

\section{Introduction}

A National Surveillance system report described eosinophilic meningitis from 0.2 to 0.3 cases/100,000 individuals in the Thai population from 2005 to $2009 .{ }^{1}$ With the higher incidence in the northern and northeastern provinces due to their eating habits, having the undercooked snail dishes is the significant risk factor. Angiostrongyliasis is one of the eosinophilic meningitis causes. And its larvae migration into the eyes is found only $1.1 \%$ of the Angiostrongyliasis cases. ${ }^{2,3}$

Angiostrongylus spp. is a rat lungworm. Humans are the accidental hosts that are infected by having this nematode in the raw intermediate hosts, such as fish, frogs, snails and prawns. ${ }^{4}$

Following entry into the human digestive system, the larvae penetrate through the blood vessels and migrate through the bloodstream to reach the central nervous system, leading to meningitis. And then the larvae can involve the orbit and other cranial nerves, especially long track nerves such as optic nerve, trigeminal nerve, abducens nerve and facial nerve as well. ${ }^{5}$

Ocular angiostrongyliasis can cause extraocular and intraocular abnormalities. The helminth can penetrate into the subconjunctival space, the extraocular muscle outside the 
eyeball. It can also be found in the anterior chamber space, the intravitreous space and the subretinal space. The helminth can induce various levels of the inflammation depending on its location and cause numerous ocular complications such as uveitis, glaucoma, retinitis, optic neuritis, and strabismus. ${ }^{6}$

\section{Case Report}

A 47-year-old Thai female was referred to Prasat Neurological Institute of Thailand with a severe headache. She reported usually eating raw fish and snails. She was later diagnosed with eosinophilic meningitis. After she has got headache for 8 days, she complained about visual loss in her right eye, which had decreased to hand motions. No inflammation was seen in the anterior segment of that eye. A relative afferent pupillary defect was weakly presented in her right eye.

From the fundus examination, we found the Angiostrongylus larva in the subretinal space in the inferotemporal quadrant with massive macular edema (figure 1). We applied the focal photocoagulation laser to the living larva to immobilize it and to the retinal tissue surrounding the parasite to prevent further migration (figure 2). The patient was treated with oral albendazole for 21 days combined with oral prednisolone for 1 month. Although the immunoblotting technique was negative for Angiostrongylus cantonensis, we identified the Angiostrongylus larvae based on its physical characteristics. A stool examination was also negative for oocyte and larva. Eosinophilia was detected in the serum and cerebrospinal fluid.

After treatment, the extensive macular edema had atrophic changes in all retinal layers.

The dead larva remained in the retina surrounded by the laser scar. Her vision did not recover. The final visual acuity in her right eye was counting fingers.

\section{Discussion}

In patients with the eosinophilic meningitis from Angiostrongylus spp, only $1.1 \%$ had ocular involvement. $^{2}$ Ocular angiostrongyliasis can be presented simultaneously with eosinophilic meningitis or after the eosinophilic meningitis. ${ }^{7,8}$ The incubation period of ocular angiostrongyliasis is between 2 weeks and 2 months after snail ingestion. ${ }^{2,7}$

The helminth can migrate to the orbit by traveling between the optic nerve and its sheath then penetrate the

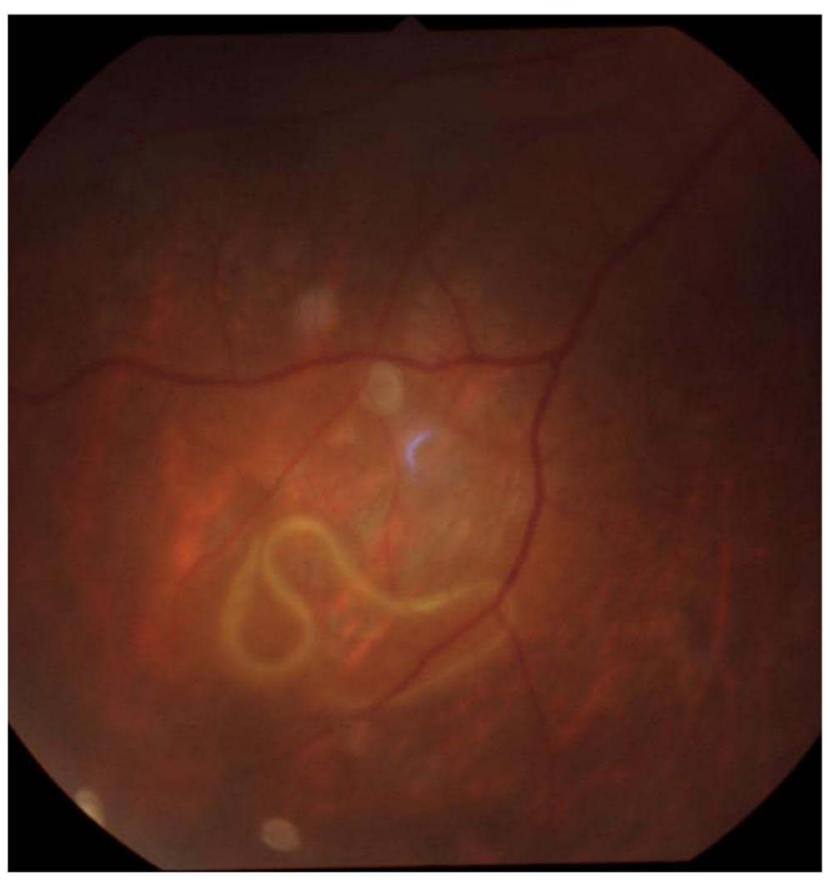

Figure I The subretinal larva in the inferotemporal quadrant.

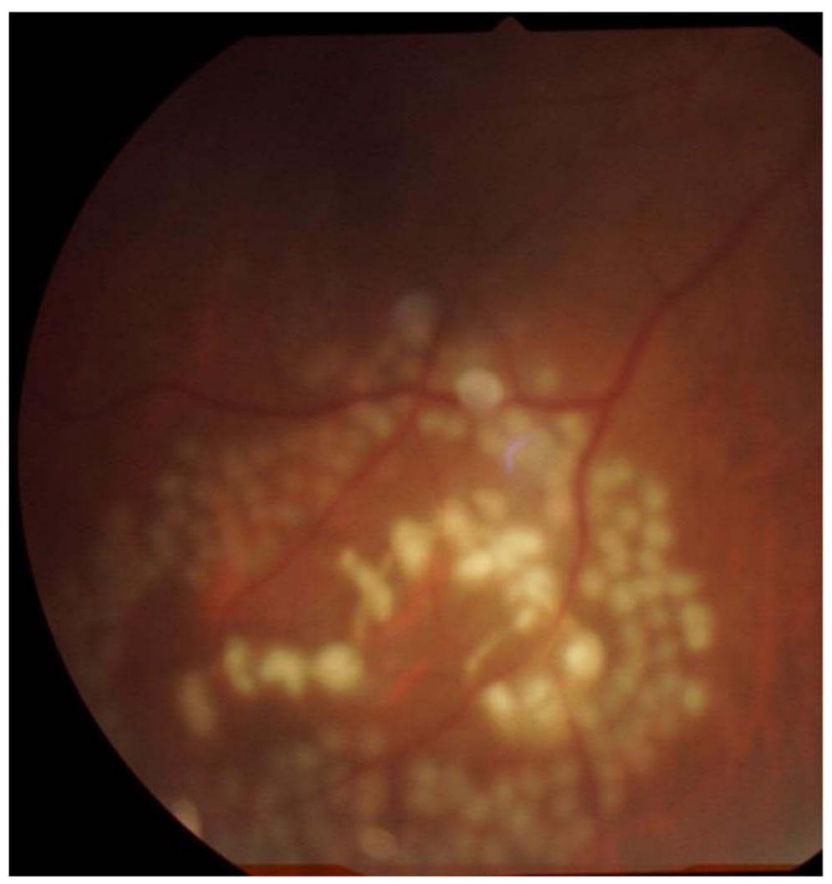

Figure 2 The subretinal larva after application of focal photocoagulation laser on and around the larval body.

eye at the cribriform plate. ${ }^{9}$ Another hypothesis is that the larva can reach the eyeball via the bloodstream. ${ }^{3}$

The most common site of ocular angiostrongyliasis is in the intravitreous space. ${ }^{6}$ However, the presence of helminth in the subretinal space is very rare. In the previous 
studies that reported treatment of the subretinal larvae, the authors used focal laser photocoagulation combined with anti-helminthic drug and systemic steroid administration. ${ }^{8}$ Most of their subretinal larval cases had poor final visual outcomes without the specification of the retinal area where the helminth was found. ${ }^{8}$

Patients generally present with poor initial visual acuity with retinal abnormalities such as macular edema, serous retinal detachment, and other retinal pigment epithelial alterations. Only one of them had good initial vision with the subretinal track, the only one evidence of subretinal larval penetration in this patient. Without any permanent retinal damage or retinal pigment epithelial alteration, her vision remained the same following the treatment. ${ }^{2,8}$

We can predict the final visual outcome from the initial visual acuity and the retinal pathology.

If patients present with macular edema or serous retinal detachment, the final vision will be poor. ${ }^{2,8}$ The retinal edema which turn to chorioretinal atrophy may be caused by the toxin secreted from larva even after its eradication.

As in the brain, the death of larvae can simultaneously produce catastrophic effects from toxic substances released by the necrotic parasites. ${ }^{10}$ Despite eradication of the parasite and no new occurrence of an infestation, the inflammatory process in the chorioretinal layers may persist or progress. So the retinal lesion might turn to atrophic scar instead of normal tissue.

The inflammation can also result from an immune response. As in the previous report, the patient with eosinophilic meningitis had necrotizing retinitis bilaterally. Although no larva was detected in the eyes, a Western blot test was positive for Angiostrongylus cantanensis in the subretinal fluid and serum. An enzyme-linked immunosorbent assay of the cerebrospinal fluid and serum also was positive. The authors hypothesized that beside the inflammatory response in the retina that can be caused directly by the larva, the inflammation may occur as the result of a host immune reaction to the parasite meningitis without ocular penetration. ${ }^{11}$

The chorioretinal inflammation occurs from either the immune response or direct toxicity released from the larva; only parasitic eradication and systemic steroid administration are insufficient to prevent permanent tissue damage in the chorioretinal layers. An intravitreous anti-vascular endothelial growth factor or local steroid injection should be considered to decrease the inflammation in the chorioretinal layers and possibly prevent the atrophic change and preserve the vision.

\section{Conclusion}

Although the subretinal angiostrongyliasis is a very rare condition, once it occurs the vision of that eye will end up with low vision or blindness. Because of the massive inflammation induced by the helminth in the retina, the earlier the inflammation is controlled, the higher the rate is of visual improvement that can be expected. If the inflammation can be stopped in time, the chorioretinal atrophy will not occur, and then, we may prevent the permanent visual loss. Local administration of high potency antiinflammatory drugs should be considered.

\section{Ethic and Consent}

The patient gave written informed consent to the publication of this case report and its accompanying photographs. This case report was reviewed by the Neurological Institute of Thailand Research and Ethics Committee and was granted approval to proceed.

\section{Acknowledgments}

I would like to thank Dr. Niphon Chirapapaisan for valuable advice.

\section{Disclosure}

The author has no conflicts of interest related to this work.

\section{References}

1. Eamsobhana P. Angiostrongyliasis in Thailand: epidemiology and laboratory investigations. Hawaii J Med Public Health. 2013;72(6 Suppl 2):28-32.

2. Sawanyawisuth K, Kitthaweesin K, Limpawattana P, et al. Intraocular angiostrongyliasis: clinical findings, treatments and outcomes. Trans $R$ Soc Trop Med Hyg. 2007;101(5):497-501. doi:10.1016/j. trstmh.2006.07.010

3. Andrade GC, Dias JRO, Maia A, et al. Intravitreal Angiostrongylus cantonensis: first case report in South America. Arq Bras Oftalmol. 2018;81(1):63-65. doi:10.5935/0004-2749.20180014

4. Sinawat S, Yospaiboon Y, Sinawat S. Subretinal angiostrongyliasis-induced optic neuritis. Clin Ophthalmol. 2013;7:977-979. doi:10.2147/OPTH.S43565

5. Punyagupta $S$, Juttijudata $P$, Bunnag $T$. Eosinophilic meningitis in Thailand. Clinical studies of 484 typical cases probably caused by Angiostrongylus cantonensis. Am J Trop Med Hyg. 1975;24(6 Pt 1):921-931. doi:10.4269/ajtmh.1975.24.921

6. Diao Z, Wang J, Qi H, Li X, Zheng X, Yin C. Human ocular angiostrongyliasis: a literature review. Trop Doct. 2011;41(2):76-78. doi:10.1258/td.2010.100294

7. Sinawat S, Sanguansak T, Angkawinijwong T, et al. Ocular angiostrongyliasis: clinical study of three cases. Eye. 2008;22 (11):1446-1448. doi:10.1038/eye.2008.135

8. Sinawat S, Trisakul T, Choi S, Morley M, Sinawat S, Yospaiboon Y Ocular angiostrongyliasis in Thailand: a retrospective analysis over two decades. Clin Ophthalmol. 2019;13:1027-1031. doi:10.2147/ OPTH.S204380 
9. Teekhasaenee C, Ritch R, Kanchanaranya C. Ocular parasitic infection in Thailand. Rev Infect Dis. 1986;8(3):350-356. doi:10.1093/ clinids/8.3.350

10. Cuckler AC, Egerton JR, Alicata JE. Therapeutic effect of thiabendazole on Angiostrongylus cantonensis infections in rats. J Parasitol. 1965;51(3):392-396. doi:10.2307/3275961
11. Liu IH, Chung YM, Chen SJ, Cho WL. Necrotizing retinitis induced by Angiostrongylus cantonensis. Am J Ophthalmol. 2006;141 (3):577-579. doi:10.1016/j.ajo.2005.09.033

\section{Publish your work in this journal}

The International Medical Case Reports Journal is an international, peer-reviewed open-access journal publishing original case reports from all medical specialties. Previously unpublished medical posters are also accepted relating to any area of clinical or preclinical science. Submissions should not normally exceed 2,000 words or 4 published pages including figures, diagrams and references. The manuscript management system is completely online and includes a very quick and fair peer-review system, which is all easy to use. Visit http://www.dovepress.com/testimonials.php to read real quotes from published authors. 